\title{
A method to optimize intermediate-passband photometric systems
}

\author{
P. Royer ${ }^{\star}$ and J. Manfroid ${ }^{\star \star}$ \\ Institut d'Astrophysique, Université de Liège, avenue de Cointe 5, B-4000 Liège, Belgium \\ Received January 2; accepted March 25, 1996
}

\begin{abstract}
A method is proposed to optimize the definition of existing photometric filters in order to minimize passband-matching problems. It is argued that the "improved" systems should generally yield better astrophysical parameters than the original ones. Although of a general scope, the method is checked on intermediate-passband filters of the Strömgren system.
\end{abstract}

Key words: methods: data analysis — methods: observational — techniques: photometric

\section{Introduction}

It is widely recognized that the use of non-standard filters is a major source of photometric errors. Color transformation procedures are unable to accurately correct these deviations. For a discussion of these errors, see e.g., Garrison (1985), Cousins \& Jones (1976), Manfroid \& Sterken (1987, 1991, 1992), Sterken \& Manfroid (1987), Young (1974, 1988, 1992), Wildey (1992).

Reproducing the standard systems is no easy task as can be judged from the filter profiles published in the users' manual of various observatories.

One expects the impact of passband mismatch to depend on the characteristics of the standard filters, e.g., a $1 \mathrm{~nm}$ error on the central wavelength of a narrow $550 \mathrm{~nm}$ filter is certainly less damaging than the same error on a similar filter centered at $\mathrm{H} \beta$. Some photometric bands such as those used for measuring the $\beta$ index were adopted with obvious constraints in view, but others bands were defined rather empirically, and could have been chosen differently — within reasonable limits - for serving the same astrophysical purposes.

In this paper we present a method which "improves" the standard passbands of existing photometric filters by minimizing the colour-transformation problems.

\section{A transformability criterion}

The color-transformation problem has no general solution (see, e.g., Sterken \& Manfroid 1992). Existing photomet-

Send offprint requests to: J. Manfroid

${ }^{\star}$ Research Associate, Belgian Concerted Research Action (ARC)

${ }^{\star \star}$ Research Director, Belgian Fund for Scientific Research (FNRS) ric systems do not adequately sample the spectra, hence they do not provide the necessary information to estimate what magnitudes will be obtained when a filter is modified. This sampling problem has been addressed in detail by Young (1974, 1988, 1992-1994), who has shown how to design a "transformable" system by adjusting the central wavelength and profile of the bands and by using a sufficient number of bands so as to satisfy the sampling theorem.

A closely related approach to solve the "local" transformability problem was used by Manfroid (1985a, b) who, as proposed by Young (1974), doubled the number of filters by adding a second set of passbands at slightly different wavelengths, essentially increasing the sampling where it matters most in the case of system mismatches. This achieves a compromise which has been further studied theoretically by Wildey (1992), who confirmed that the method can be very effective in eliminating transformation errors.

The relative success of the usual colour-transformation procedures in most photometric works is due to the fact that stellar spectra form families of functions - as shown by the well-known sequences in colour diagrams. Stellar spectra are not arbitrary functions and satisfying the sampling theorem may be unnecessary when only selected types of stars are studied.

The very existence of relations between spectra of the same family induces the existence of a color transformation for that family, so high-quality observations of a few well-selected standards - within that family! - may yield a satisfactory transformation (see, e.g., Bessel 1983; Olsen 1983; Lynga \& Wramdemark 1984; Manfroid 1985a, b; Cousins 1986). 
Problems arise when stars of various types are mixed, since the relations are not the same and are quite incompatible. Manfroid (1992) showed that simple physical constraints cannot be respected when different families are merged. He showed that this concept of families is very strict: for instance, multiple stars of a same family cannot be included in that family. In such cases, only an adequate sampling of the spectra would allow correct color transformations.

The bands of most photometric systems were chosen for astrophysical reasons, such as isolating a spectral feature. Technical aspects also played a role (e.g., in the Walraven system). The older, wide-band systems were rather poorly defined, and simply made use of available filters. It is then very likely that in many cases, a slight modification of the definition of the passbands could improve the astrophysical interest of many a photometric system.

Let us consider the multidimensional space $P$ of the passband characteristics (e.g., central wavelength, FWHM and various moments). Now, imagine we want to build some ideal function over this space, representing the astrophysical interest of the passband. This is certainly impossible for a single band. Several bands (say, $m$ ) must be considered together - and the dimensionality of $P$ should be multiplied accordingly (let us call this space $P^{m}$ ). The astrophysical interest could be measured by the quality of the relations that can be derived between the $m$ colour indices (magnitudes) and $k$ fundamental physical characteristics $\left(T_{\text {eff }}, \log g\right.$, etc.) best defining the stars. (of course we must have $m \geq k$ ). It would then be possible to locate maxima of this function over $P^{m}$ and, thus, to define some ideal photometric systems. These maxima are probably located close to existing passbands, and could be used to improve the definition of these systems. Of course, it is not easy to build such a function, which would be very different according to the stars being considered (the same star families as above). However, it is quite natural to assume that these maxima correspond to sites of optimal transformability : moving around these sites in $P^{m}$ yields the smallest degradation in the relations between colour indices (magnitudes) and physical parameters. This should also lead to the smallest changes in the transformation relations and, presumably in their quality.

A "transformability function" can be calculated by performing synthetic photometry over families of stars, with several sets of passbands within a significant domain in space $P^{m}$. The simplest realistic cases involve 3 or 4 indices/magnitudes and 2 or 3 bandpass characteristics, which rapidly leads to overwhelming calculation and representation problems. However, considering that the bandpasses of existing systems are probably not too far from ideal, it is quite acceptable to study each band separately, hence to go back to space $P$.
Let us compare the values of the colour index CI at $\boldsymbol{p}=\left(p_{1}, \ldots, p_{n}\right)$ and at nearby positions $\boldsymbol{p}_{i}=\boldsymbol{p}+$ $\mathrm{d} \boldsymbol{p}_{i}=\left(p_{1}, \ldots, p_{i}+\mathrm{d} p_{i}, \ldots, p_{n}\right)(i=1, \ldots, n)$ in the $n$ dimensional space $P$ of the parameters corresponding to one of the bands involved in the definition of this particular index. The calculations are carried out for a whole set of stars. Let us denote by $\phi_{i}(\boldsymbol{p})$ the rms residual resulting from a linear transformation (with CI or, possibly, other indices as the independent variables) between the values of CI obtained before and after a modification $\mathrm{d} \boldsymbol{p}_{i}$. Function $\phi_{i}(\boldsymbol{p})$ represents the error occuring during local color transformation corresponding to parameter $i$. Mathematically, it is the opposite of a tranformability criterion, i.e., it is a measure of the 'untransformability' of this passband.

Each dimension is treated independently, i.e., the untransformability along axis $i$ is checked for every set of parameters $p_{j}, j \neq i$. More generally, the functions $\phi_{i}(\boldsymbol{p})$ form a $n$-dimensional vector field which completely describes the local untransformability.

We can define a scalar untransformability function as $T(\boldsymbol{p})=\sqrt{\sum_{i=1}^{n} a_{i} \phi_{i}^{2}(\boldsymbol{p})}$. where the weights $a_{i}$ reflect the relative importance we want to give to the various passband characteristics.

There is some arbitrariness in this definition, since the increments $\mathrm{d} \boldsymbol{p}_{i}$ can be defined in various ways. Steps in central wavelengths or FWHM can be constant, or wavelength dependent. Increments in higher-order moments, or in any additional characteristics of the passbands are equally difficult to define. The logical point of view is that every additional constraint on these definitions should reflect the diversity of the filter sets actually found in the observatories, i.e., presumably, the difficulties encountered by the manufacturers to respect the nominal characteristics.

\section{An application to Strömgren passbands}

We limit ourselves to a 2 -D space $P$ with the central wavelength $\lambda$ as one coordinate and the FWHM $w$ as the second coordinate. Spectra are taken from the grid of synthetic spectra calculated by Kurucz (see Buser \& Kurucz 1992) because they form a homogeneous, and rather complete data set. Other spectral databases (Jacoby et al. 1984; Silva \& Cornell 1992) were studied, mainly for checking purposes; they yielded equivalent, though less complete results, and we shall not discuss them further.

Colour transformations are computed between successive points on a grid $\boldsymbol{p}=(\lambda, w)$ and $\boldsymbol{p}_{1}=\boldsymbol{p}+\mathrm{d} \boldsymbol{p}_{1}=$ $(\lambda+\mathrm{d} \lambda, w)$ or $\boldsymbol{p}_{2}=\boldsymbol{p}+\mathrm{d} \boldsymbol{p}_{2}=(\lambda, w+\mathrm{d} w)$.

We first consider each dimension independently, i.e., the (un)transformability along the $\lambda$ axis is checked for every $w$, and conversely.

We choose to define constant $\mathrm{d} \lambda$ increments of $1 \mathrm{~nm}$. The range of wavelengths is small compared to the central wavelength, so this choice is not very critical. In $w$, we explored changes over a factor of 2 , approximately centered 
on the FWHM of the standard passband, and we used proportional increments, $\mathrm{d} w / w=k$. The value of $k$ is such that 30 steps cover the entire domain.

Two stellar families were considered, which correspond to major zones of application of the Strömgren system, and which are known to need distinct colour transformations.

\subsection{Early-type stars}

Figures 1a-c show $\phi_{\lambda}$ as a function of $\lambda$, plotted for different value of $w$, in the neighbourhood of the nominal passbands of the $v, b$ and $y$ filters respectively. The data include stars of spectral types $\mathrm{O}$ to $\mathrm{A}$, gravities $\log g=4.5$ and solar metallicities $(c=0.0)$.

Two general remarks can be made about these figures:

- The smallness of $\phi_{\lambda}(\boldsymbol{p})$ results from the use of synthetic spectra in the calculations: the transition from one spectrum to its neighbours (in the model grid) is very smooth. Calculations based on catalogs of observed spectra show qualitatively similar results - because the spectra are, of course, taken from the same family of stars - but with somewhat larger $\phi_{i}(\boldsymbol{p})$. This is largely due to observational errors giving larger inter-spectra scatter which degrades the family relationship and, thus, yields poorly defined colour transformations.

- They show that the narrow filters are generally more sensitive to a change in central position $(\mathrm{d} \lambda)$ than the wider ones. We will see that this is not true for the change in width $(\mathrm{d} w)$.

The $v$ filter is centered on $\mathrm{H} \delta$ at $410 \mathrm{~nm}$ (Fig. 1a). Excepted for the widest passbands, there are 3 minima. One of them can be attributed to $\mathrm{H} \delta$. The minimum at about $434 \mathrm{~nm}$ is due to $\mathrm{H} \gamma$, and the third one (419 nm) is roughly halfway in between. The $\mathrm{H}$ lines correspond to minima because moving a filter away from these positions increases the flux in one wing and decreases it in the other, so the system is quite "blind" to such a variation. The third minimum is not located exactly in the middle of the interval between $\mathrm{H} \gamma$ and $\mathrm{H} \delta$ because $\mathrm{H} \gamma$ has a greater equivalent width than $\mathrm{H} \delta$ : it is representative of an almost line-free spectral domain where the passband has little overlap with these hydrogen lines.

With regard to central wavelength variations only, this position seems very attractive for the $v$ filter:

- it's a minimum in $\phi_{\lambda}(\boldsymbol{p})$ for the narrow filters and, for the standard width, $\phi_{\lambda}(\boldsymbol{p})$ is hardly larger there than at nominal position;

- the corresponding passband is almost free of lines, so $v$ magnitudes can be used directly: corrections for $\mathrm{H} \delta$ are no longer necessary, which means a gain in accuracy;

- the filter would gather more flux there, so the precision and the limiting magnitudes would be increased.
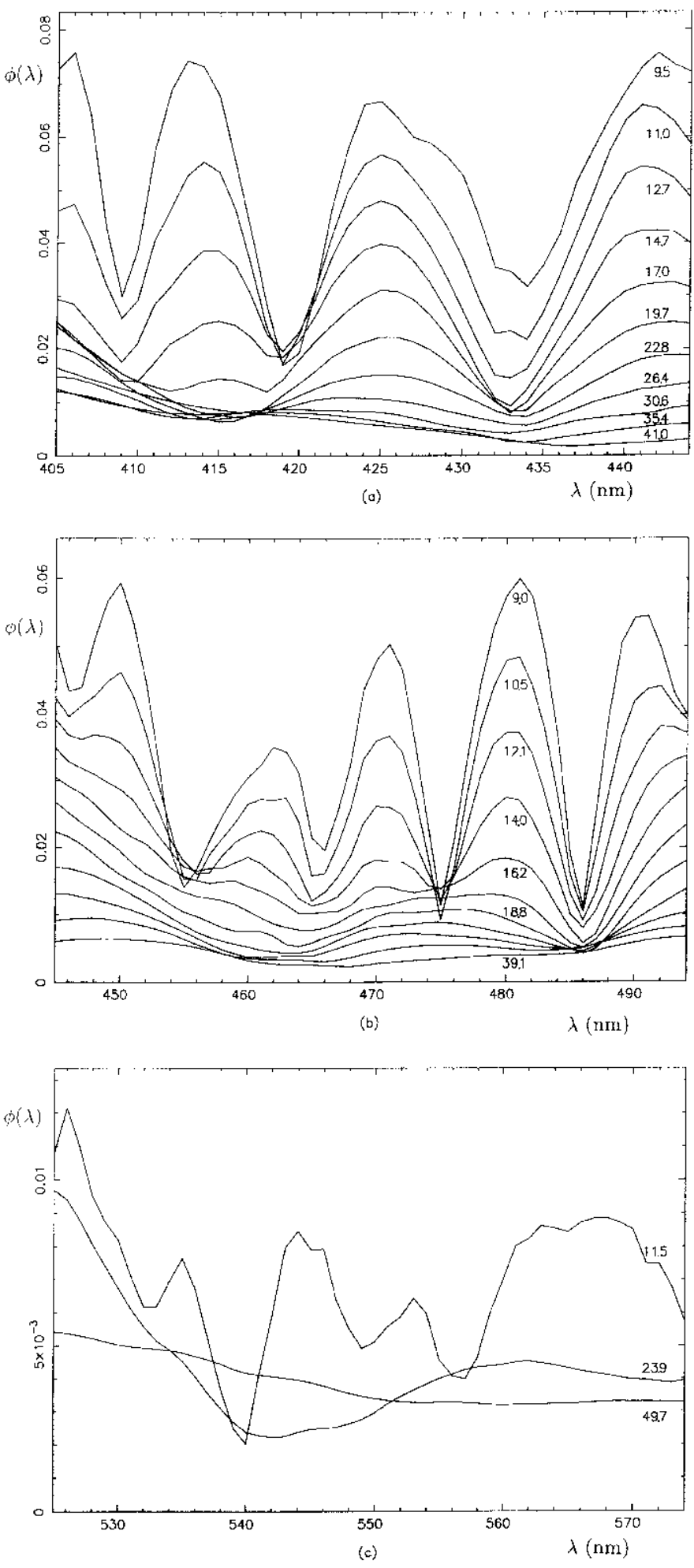

Fig. 1. Untransformability $\phi_{\lambda}$ (in units of $0.01 \mathrm{mag}$ ) relative to passbands $v, b$ and $y$ (from top down) as a function of $\lambda$, for different values of $w$ in the case of $\mathrm{O}-\mathrm{A}$ stars. The different curves are labeled with their corresponding FWHM. The FWHM of the standard passbands is 19.7, 18.8 and $23.9 \mathrm{~nm}$ respectively, their central wavelength corresponds to $410 \mathrm{~nm}$ for $v, 470 \mathrm{~nm}$ for $b$ and $550 \mathrm{~nm}$ for $y$ 


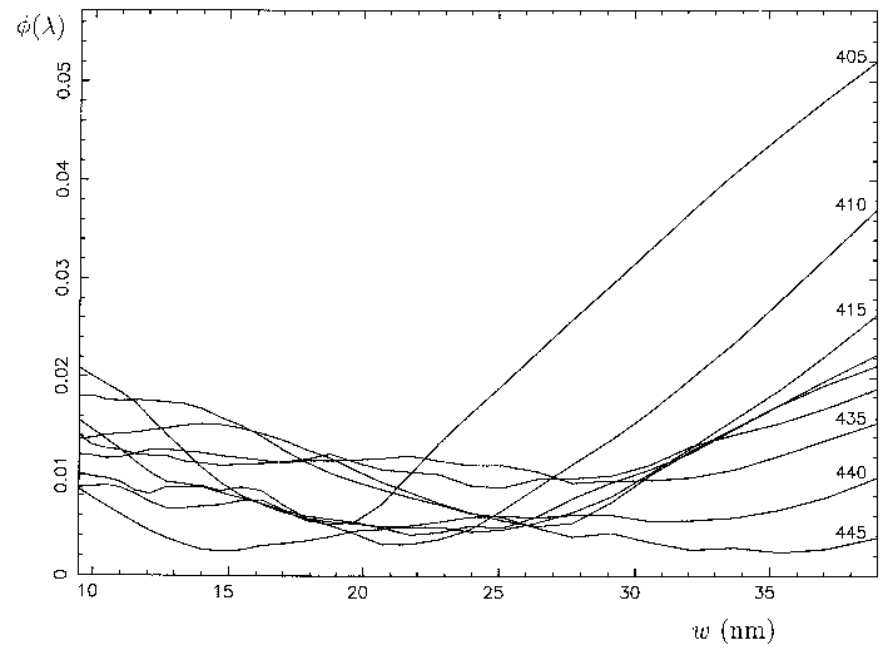

Fig. 2. Untransformability $\phi_{w}$ relative to passband $v$ as a function of $w$, for different values of $\lambda$ in the case of $\mathrm{O}-\mathrm{A}$ stars

Figure $1 b$ shows a whole series of minima for the narrow filters but only two for the standard-width ones, the nearest being at $464 \mathrm{~nm}$, i.e., $6 \mathrm{~nm}$ to the blue of the standard position $(470 \mathrm{~nm})$.

For the sake of readability, Fig. 1c shows only three curves relative to, respectively, the narrowest, the standard-width and the widest filters. We see that a standard-width filter would benefit from a 6 to $10 \mathrm{~nm}$ shift toward the blue; however, the improvement is rather slight, and the present standard filter is not badly placed. Only for a much narrower filter would it be really interesting to center it at $540 \mathrm{~nm}$ instead of $550 \mathrm{~nm}$.

Figure 2 shows $\phi_{w}$ as a function of $w$, for different $\lambda$, in the case of $v$. Excepted for the appearance of characteristics due to the Balmer discontinuity in the curves concerning the bluest positions (curves with lowest labels), there are no obvious features. So, to give a better general idea, we plotted the overall untransformability function, defined as the modulus $T(\boldsymbol{p})=\sqrt{a_{1} \phi_{1}^{2}(\boldsymbol{p})+a_{2} \phi_{2}^{2}(\boldsymbol{p})}$, in Figs. 3 and 4 , still in the case of $v$, with 2 additional weighting schemes between the $\lambda$ and $w$ effects (with $a_{1}=1$ and $a_{2}=1,2$ or 4 ). This is approximately equivalent to increasing $d w$ by factors of 2 and 4 .

Along the sequence of 3 -D plots, we clearly see that, as expected, the importance of the maxima due to the sensitivity of narrow filters to a $\mathrm{d} \lambda$ change decreases progressively as the relative importance of the sensitivity to $\mathrm{d} w$ increases. Giving a strong weight $a_{2}$ to the FWHM effects, can even lead to a maximum replacing a minimum. This can occur because of the increased FWHM sensitivity of a filter centered on a strong line.

In the contour plot, we see that some minima remain at the same approximate location of the 2-dimensional space of filter characteristics whatever the weight given to either of these characteristics.
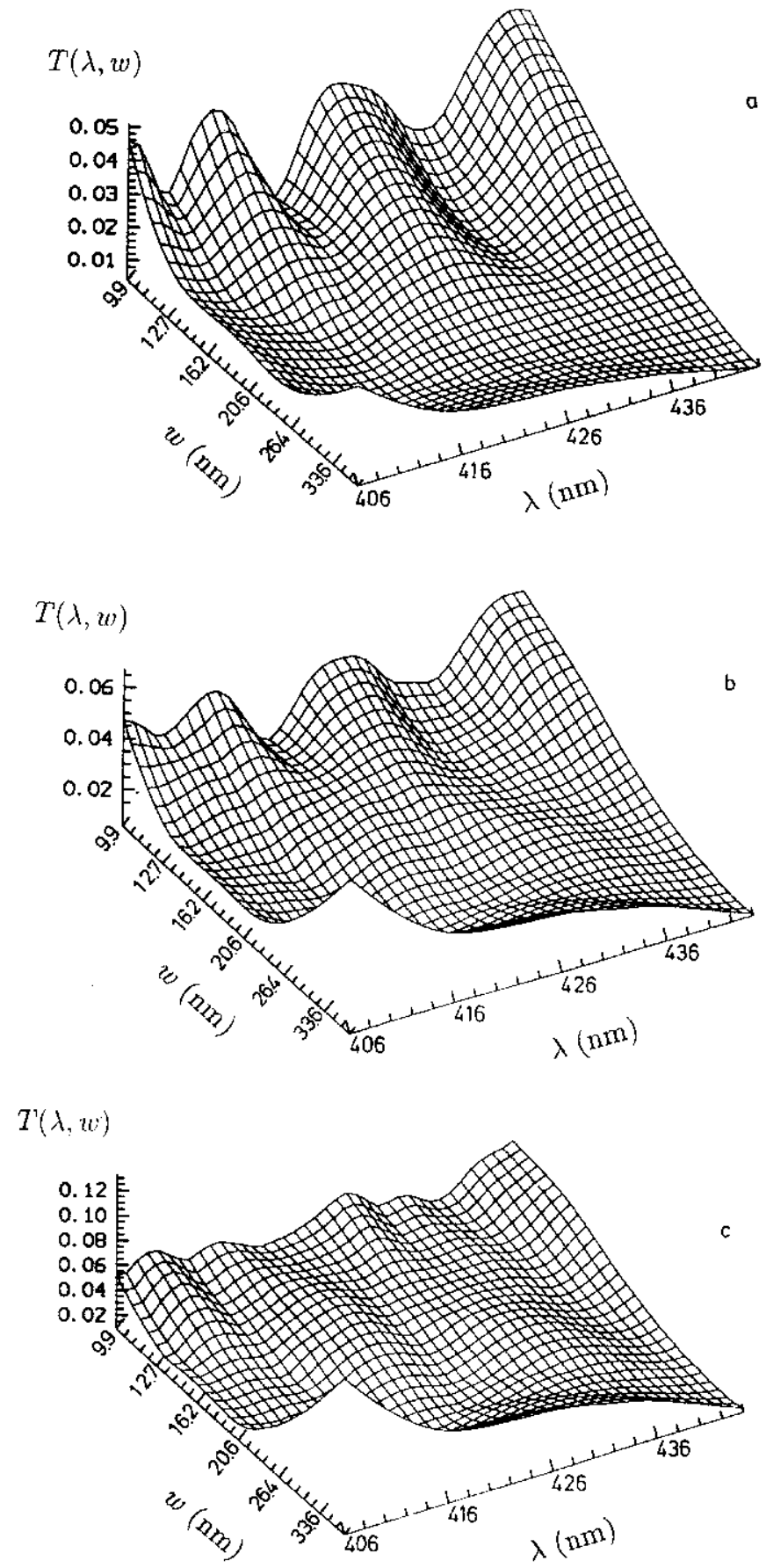

Fig. 3. Untransformability function $T(\lambda, w)$ relative to passband $v$ in the $(\lambda, w)$ plane in the case of $\mathrm{O}-\mathrm{A}$ stars. Top: steps in $\mathrm{d} \lambda$ and $\mathrm{d} w$ are as defined in the text $\left(a_{1}=a_{2}=1\right)$. Middle: steps $d w$ have been increased by a factor $2\left(a_{2}=2\right)$. Bottom: steps $\mathrm{d} w$ have been increased by a factor 4 relatively to the upper plot $\left(a_{2}=4\right)$. The standard passbands lie in the positions specified in the caption of Fig. 1 


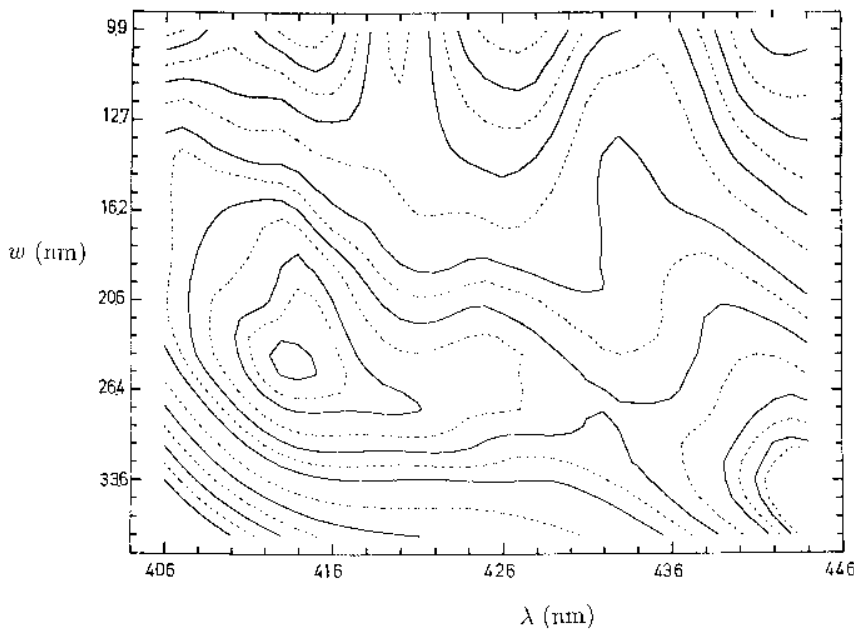

Fig. 4. Contour plot of the untransformability function $T(\lambda, w)$ presented in the central plot of Fig. 3

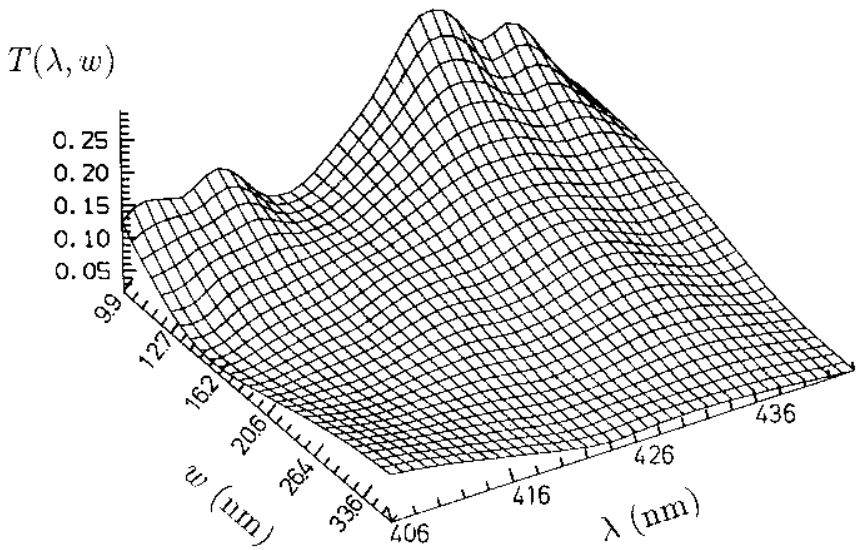

Fig. 5. Untransformability function $T(\lambda, w)$ relative to passband $v$ in the $(\lambda, w)$ plane for $\mathrm{A}-\mathrm{G}$ stars $\left(a_{1}=1, a_{2}=2\right)$

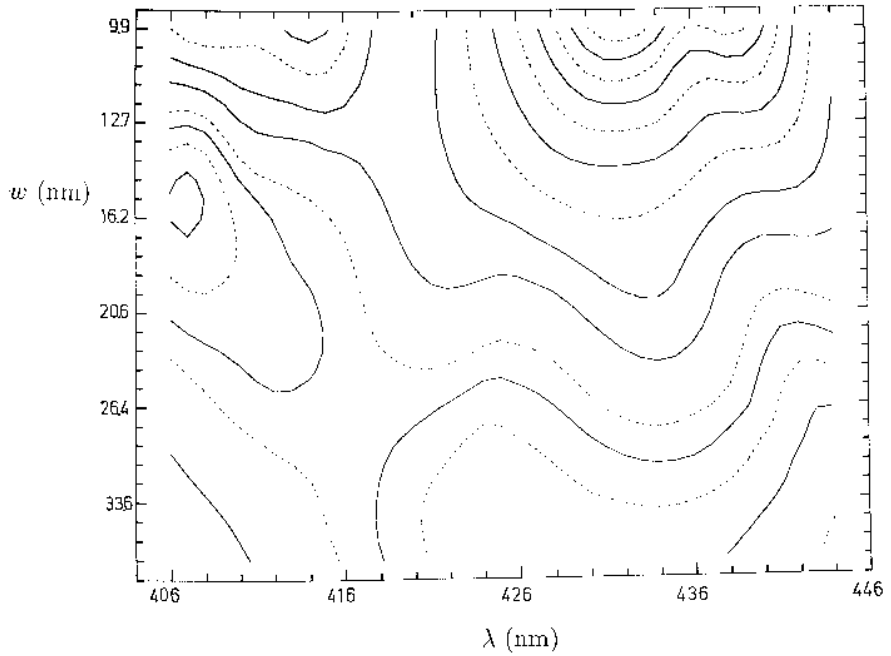

Fig. 6. Contour plot of the untransformability function $T(\lambda, w)$ presented in Fig. 5

\subsection{A-G stars}

Figure 5 deals with the $v$ passband, for cooler stars of spectral type A-G (and, still, $\log g=4.5$ and solar metallicities). Although the central minimum is less marked, we still can distinguish all three minima for the narrow filters, but we see in the corresponding contour plot of Fig. 6 that the minimum has moved away from the position it occupied in Fig. 3, hence, a single undersampled photometric system is not ideally suited to both hot and cold stars. For A-G stars, the ideal $v$ filter would be somewhat bluer and narrower than the current standard one.

Similar calculations for the other passbands lead us to the same conclusions. It must be noticed that, because of the strength and number of features to be found in the spectra, the levels of some characteristics of $T(\boldsymbol{p})$ are much higher when we treat $A-G$ stars than when we consider hotter stars, particularly in the case of $v$ and $b$.

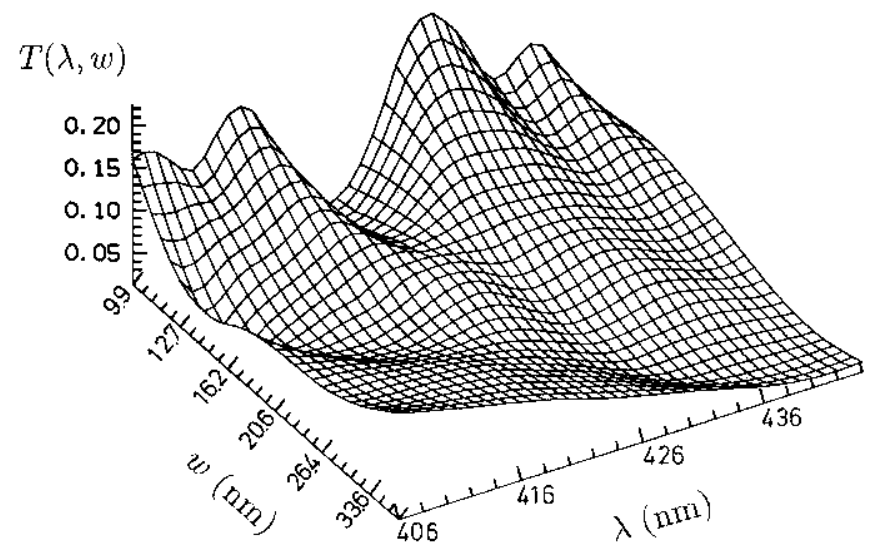

Fig. 7. Untransformability function $T(\lambda, w)$ relative to passband $v$ in the $(\lambda, w)$ plane for giant $\mathrm{A}-\mathrm{G}$ stars. $\left(a_{1}=1, a_{2}=2\right)$

\subsection{Luminosity effects}

Figure 7 concerns the $v$ filter for $\mathrm{A}-\mathrm{G}$ stars with solar metallicities, but this time with a lower surface gravity, $\log g=2.0$, characteristic of giant stars. Here, the $\mathrm{H}$ lines have smaller equivalent widths, so the central minimum is much deeper and the 2 minima corresponding to the lines are less marked. Nevertheless, the minimum of $T(\boldsymbol{p})$ still lies close to the one obtained for the A-G dwarfs. In this case, this indicates temperature to be a greater discriminant of ideal filter characteristics than surface gravity. Same conclusions hold for $b$.

Concerning $y$, the values reached by $T$ are so low as to have little effects on the ideal $y$ characteristics from the photometric point of view. Differences can be seen between dwarfs and giants but they don't justify modifying the existing passband characteristics. 


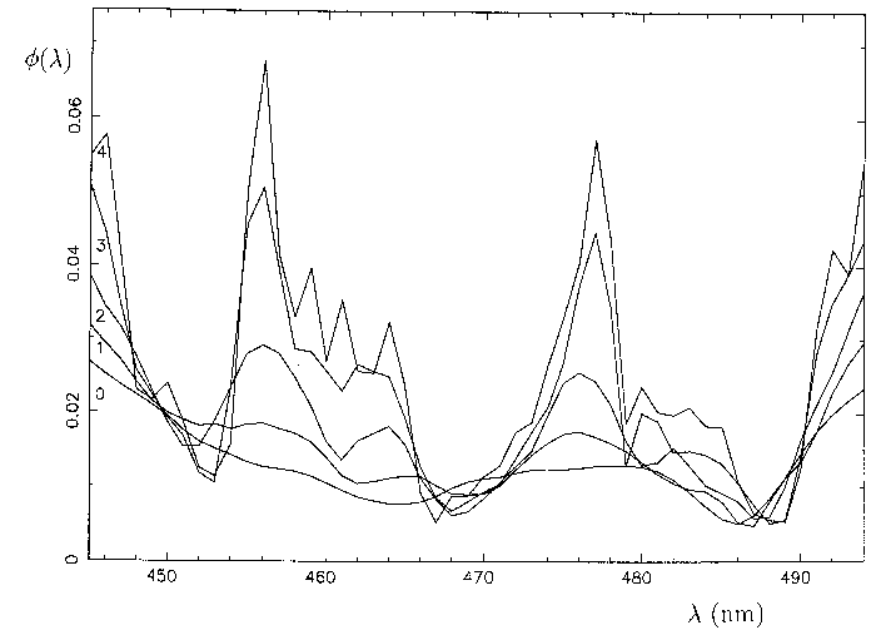

Fig. 8. Untransformability $\phi_{\lambda}$ relative to passband $b$ as a function of $\lambda$, for different values of squareness. Filter $\# 0$ is the $b$ standard; filter \#4 is rectangular. All filters have the same FWHM

\subsection{Late-type stars}

We carried out calculations on synthetic and actual spectra of later type stars. The wide diversity of stellar types and classes leads to the definition of a correspondingly large number of distinct ideal systems, which is a lessthan-ideal situation. This confirms the problems usually met when trying to get accurate color transformations for late-type stars. Quite distinct color transformations must be applied to the many classes and sub-classes of cool stars, and this is clearly not always feasible. This stresses the special need for accurately reproduced standard bandpasses whenever studying cool stars.

\subsection{Effect of high-transmission filters}

The introduction of high-transmission filters with steep profiles proved to be disastrous in terms of sensitivity to filter characteristics. This is examplified in Fig. 8 where the effect of wavelength changes is shown for different degrees of squareness of the $b$ filter.

\section{Conclusion}

The method we propose allows refining or establishing better definitions of photometric systems in terms of reduced sensitivity to poor passband matching and better astrophysical performances. While spectacular improvements are not expected in all cases, especially in wideband systems, intermediate bands are particularly sensitive to small changes in their characteristics and deserve to be carefully designed.

The untransformability function is very much dependent on the group of stars for which it is computed. This underlines the importance of clearly defining distinct families of stars in colour-transformation procedures. It also shows that the usual advice of observing standards representing the widest range of stellar types and classes must be qualified. Standard stars must belong to the same families as the programme stars (see Manfroid 1992, 1993). Other standards could still be useful for computing atmospheric extinction (Manfroid 1993), but otherwise they represent a waste of observing time, and a risk of degrading the results, if they are included in the transformation procedure. Such a degradation is apparent in the work of Bell et al. (1994): the use of a single transformation scheme for synthetic photometry performed on the entire Kurucz grid leads to erroneous interpretation as shown by Roberts \& Grebel (1995).

Hence, our calculations performed on the Strömgren system lead to the rather natural conclusion that a universal ideal photometric system does not exist. Significant gains in accuracy and precision could be achieved by refining the definition of intermediate-band systems and (see Sect. 3.2) limiting the scope of each system to very welldefined stellar types.

Acknowledgements. The computations have been performed on computers at the SEGI and at the Institut d'astrophysique (University of Liège), partly thanks to contracts ARC94/99178 "Action de recherche concertée de la Communauté française" (Belgium), and SC005 "Service centers and research networks" initiated by the Belgian State, Prime Minister Office, Science policy programming under SSTC. Partial support through the PRODEX XMM-OM project is also gratefully acknowledged.

\section{References}

Bell R.A., Paltoglou G., Tripicco M.J., 1994, MNRAS 268, 771 Bessel M.S., 1983, PASP 95, 480

Buser R., Kurucz R.L., 1992, A\&A 264, 557

Cousins A.W.J., 1986, MNASSA 45, 103

Cousins A.W.J., Jones D.H.P., 1976, Mem. R. Astron. Soc. 81, 1

Garrison R.F., 1985, in: Hayes D.S. et al. (eds.) Calibration of fundamental stellar quantities, IAU Coll. 111. Reidel. Publ. Co. Dordrecht, p. 17

Jacoby G., Hunter D., Christian C., 1984, ApJS 56, 257

Lynga G., Wramdemark S., 1984, A\&A 132, 58

Matsushima S., 1969, ApJ 154, L147

Manfroid J., 1985a, Traitement des données photométriques, University of Liège

Manfroid J., 1985b, in: Hayes D.S. et al. (eds.) Calibration of fundamental stellar quantities, IAU Coll. 111. Reidel. Publ. Co. Dordrecht, p. 495

Manfroid J., 1992, A\&A 260, 517

Manfroid J., 1993, A\&A 271, 714

Manfroid J., Sterken C., 1987, A\&AS 71, 539

Manfroid J., Sterken C., 1991, in: Davis Philip A.G. et al. (eds.)

Precision photometry. L. Davis Press, p. 139

Manfroid J., Sterken C., 1992, A\&A 258, 600

Olsen E.H., 1983, A\&AS 54, 55 
Olson R., 1974, PASP 86, 80

Roberts W.J., Grebel E.K., 1995, A\&AS 109, 313

Silva D., Cornell M., 1992, ApJS 81, 865

Sterken C., Manfroid J., 1987, Proc. 27th Liège International Astroph. Coll., 55

Sterken C., Manfroid J., 1992, Astronomical Photometry - A guide. Kluwer Academic Publishers, Dordrecht

Young A.T., 1974, in: Carleton N. (eds.) Methods of experimental physics, 12, Part A. Academic Press, New York
Young A.T., 1988, in: Borucki W.J. (eds.) Second workshop on improvements to photometry, NASA CP-10015, p. 215

Young A.T., 1992, A\&A 257, 366

Young A.T., 1993, in: Butler C.J. \& Elliott I. (eds.) Stellar photometry - Current techniques and future developments, IAU Coll. 136. Cambridge University Press, p. 80

Young A.T., 1994, A\&A 288, 683

Wildey R.L., 1992, PASP 104, 290 